Du texte à la scène : langages du théâtre

\title{
Pour une sémiologie du spectacle dramatique télévisuel : All's Well that Ends Well (BBC production)
}

Analyse de deux segments filmiques

Jean-Pierre Maquerlot

\section{OpenEdition}

Édition électronique

URL : http://journals.openedition.org/shakespeare/472

DOI : 10.4000/shakespeare.472

ISSN : 2271-6424

\section{Éditeur}

Société Française Shakespeare

Édition imprimée

Date de publication : 1 novembre 1983

Pagination : 187-204

\section{Référence électronique}

Jean-Pierre Maquerlot, « Pour une sémiologie du spectacle dramatique télévisuel : All's Well that Ends Well (BBC production) 》, Actes des congrès de la Société française Shakespeare [En ligne], 4| 1983, mis en ligne le 01 janvier 2007, consulté le 23 avril 2019. URL : http://journals.openedition.org/ shakespeare/472; DOI : 10.4000/shakespeare.472 
SOCIETE FRANÇAISE SHAKESPEARE

Actes du Congrès 1982

\section{DU TEXTE A LA SCENE : Langages du Théâtre}

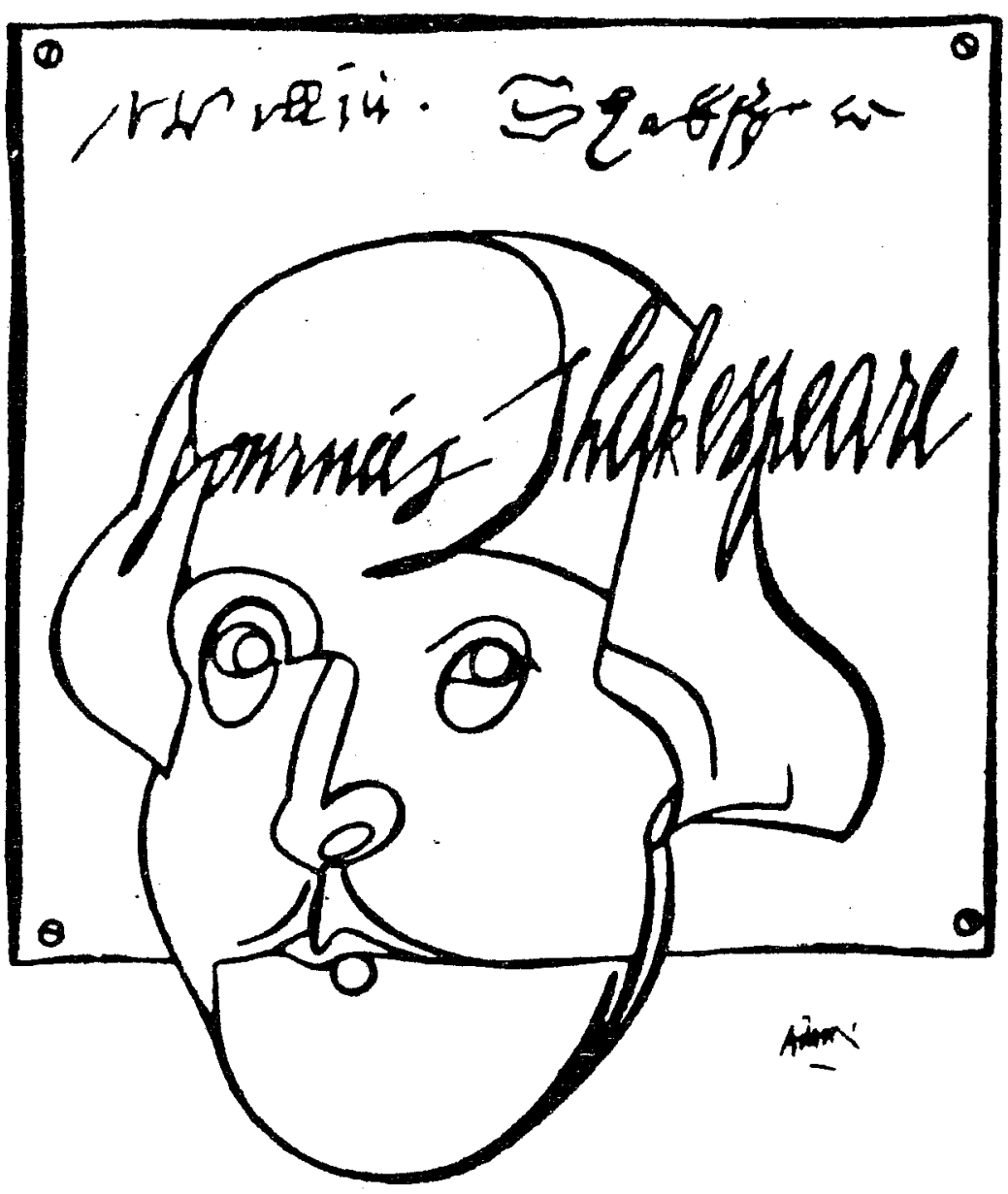

DiRecteur de la publication M.T. Jones - Davies

Publié avec le concours du Centre National de la Recherche Scientifique

JEAN TOUZOT Libraire - Editeur

38 , rue Saint-Sulpice 75278 PARIS CEDEX 061983 
Du Texte à la Scène : Langages du Théâtre.

\section{POUR UNE SEMIOLOGIE DU SPECTACLE \\ DRAMATIQUE TELEVISUEL \\ ALL'S WELL THAT ENDS WELL}

(B.B.C. production)

ANALYSE DE DEUX SEGMENTS FILMIQUES

On a beaucoup parlé dans ce colloque de mise en scène, et à juste titre. Je me propose de vous parler de mise à l'écran. Autant dire que nous quittons l'univers solide du théâtre pour celui, pelliculaire, du film. Quel film ? non pas le film de cinéma mais le film de télévision, au risque de décevoir les shakespeariens cinéphiles et de déplaire aux intellectuels téléphobes.

Pourquoi la télévision - pour la première fois, je pense - dans un Congrès de la Société Française Shakespeare ? C'est que, pour la première fois, nous sommes à la veille de disposer, avec les téléfilms de la B.B.C. déjà réalisés et ceux qui vont suivre, d'une version télévisuelle de l'intégrale des pièces de Shakespeare. «Télévisuelle» et non «télévisée», car les films en question ne visent pas à restituer à domicile des représentations théâtrales. Ils sont d'authentiques produits de télévision qu'on ne peut assimiler ni avec les adaptations proprement cinématographiques des pièces de Shakespeare ${ }^{1}$, ni avec les produits du théâtre filmé. A propos du théâtre filmé, très décrié par les amateurs de cinéma, de théâtre et de bonne télévision ${ }^{2}$, une rapide mise au point est nécessaire : même lorsqu'il se soumet le plus étroitement aux impératifs de la représentation théâtrale, le film ne renie jamais complètement sa nature cinématographique; à preuve les variations de plans, de cadrage, d'angles de prise de vue, de mouvements d'appareil qui accompagnent toujours, peu ou prou, le filmage de tout spectacle. Même les émissions télévisées qui, abusivement, prétendent nous convier «au théâtre ce soir» ne reproduisent jamais exclusivement «le point de vue du monsieur de l'orchestre $»^{3}$. Convenons toutefois que, dans la mesure où l'effet de théâtre s'obtient au détriment de l'effet de cinéma ou de télévision, ces films de repro- 
duction des spectacles dramatiques ont toute chance d'être cinématographiquement plus pauvres que les films d'adaptation. Les téléfilms de la B.B.C. appartiennent à cette dernière catégorie. A ce titre, ils peuvent servir de matériau pour qui s'intéresse aux questions que pose l'adaptation au petit écran des textes de théâtre et pour qui voudrait décrire, d'un point de vue sémiologique, le fonctionnement du spectacle dramatique télévisuel.Dans ces téléfilms se déploie une cinématographie particulière, techniquement et culturellement dépendante du medium télévisuel. C'est dire que si, à terme, le but est d'élaborer une théorie de la spécificité du langage télévisuel, il faut commencer par prendre en compte ce que nous savons déjà du langage cinématographique. Les travaux de Christian Metz sont ici d'un apport précieux; menés au contact des méthodes et des concepts de la linguistique moderne, ils ont jeté les bases d'une sémiologie du cinéma ${ }^{4}$. Si l'on peut admettre avec $\mathrm{Ch}$. Metz que cinéma et télévision sont : «deux versions technologiquement et socialement distinctes d'un même langage ${ }^{5}$, donc justiciables d'une approche méthodologique commune, l'inventaire des différences ouvre un champ de recherche nouveau. A cet égard, une étude comparative des adaptations cinématographiques des pièces de Shakespeare et des téléfilms shakespeariens de la B.B.C. peut apporter beaucoup, à condition qu'elle ne se cantonne pas à une analyse filmique, mais qu'elle aborde les questions touchant à la fonction socioculturelle du cinéma et de la télévision.

Plus modestement, je voudrais, sur deux exemples, donner à voir le travail d'un réalisateur de télévision aux prises avec un texte shakespearien. Les deux segments filmiques étudiés sont extraits d'All's Well That Ends Well réalisé par Elyah Moshinsky pour la B.B.C. en 1980. Un mot sur ce choix qui peut paraître déconcertant. Pour une première tentative d'analyse filmique, je ne voulais pas me faire la partie trop belle; je voulais résister à la tentation d'aller voir du côté des pièces historiques, du côté de Hamlet, du Roi Lear qui ont un passé cinéma- 
tographique glorieux, ou du côté du Songe d'une nuit d'été ou de la Tempête qui me semblaient se prêter mieux que d'autres pièces à un traitement cinématographique. Il était curieux de voir ce que la télévision pouvait faire d'un texte qui, à la lecture, semble assez pauvre d'atmosphère, très modérément comique et très modérément pathétique, une pièce dont les épisodes se situent en un petit nombre de lieux faiblement caractérisés. C'est cette impression de faible relief et de relative grisaille ressentie à la lecture du texte puis démentie à la vue du film qui détermina mon choix. Deux méthodes se proposaient.

- On pouvait étudier chacune des cinq séries parallèles qui composent la chaîne filmique : images, paroles, mentions écrites (en l'occurrence, les sous-titres), musique et bruits. Encore fallait-il, une fois ce travail terminé, changer de point de vue et passer de l'axe des consécutions à l'axe des simultanéités puisque le cinéma - comme le théâtre - met en oeuvre une double syntagmatique, une syntagmatique «horizontale» de la consécution et une syntagmatique «verticale» de la simultanéité. Cela impliquait que, dans un deuxième temps, l'on étudiât la coprésence des objets qui composent l'image ainsi que les combinaisons (syntagmes verticaux) entre les éléments relevant de séries différentes mais perçus synchroniquement : les rapports paroles/images, images/musique, paroles/musique etc.

- L'autre méthode, moins systématique, moins rigoureuse au regard des exigences d'une bonne sémiologie, avait l'avantage d'être plus rapide; c'est elle que je retiens parce que la seule compatible avec la durée de l'exposé. Elle consiste à saisir d'emblée le texte filmique ${ }^{6}$ comme un tout, composé d'éléments hétérogènes. Dans cette perspective, on tente de repérer directement les agencements consécutifs et simultanés, ce qui entraîne, je le reconnais, un va-et-vient assez inconfortable entre le point de vue synchronique et le point de vue diachronique. Pour ne pas alourdir l'analyse, j'ai fait abstraction du sous-titrage et des problèmes qui s'y ratta- 
chent : les rapports du sous-titre à l'image, du sous-titre à l'énonciation orale du discours, du sous-titre au signifié du discours, cette dernière question rejoignant le problème plus général du transcodage (adaptation ou traduction d'une langue à une autre).

\section{Etude du premier segment filmique : le monologue d'Helena (I.i. 76-103,éd. Arden)}

Ce segment comporte deux plans situés respectivement avant et après l'entrée de Parolles ${ }^{7}$. Nous avons ici une séquence au sens ordinaire du terme ${ }^{8}$; séquence minimale, puisqu'il faut au moins deux plans successifs pour faire une séquence; séquence linéaire puisqu'aucune interruption diégétique ne vient scinder le cours de la narration filmique. La première question qui se pose est celle-ci : pourquoi une séquence plutôt qu'un seul plan autonome pour filmer cet épisode ? Le découpage en deux plans témoigne de la fidélité du réalisateur au texte shakespearien. En effet, l'entrée de Parolles opère une coupure dans le signifié global du monologue : on passe d'une méditation introspective au portrait moral du nouvel arrivant; à cette coupure du signifié textuel correspond la coupure du signifiant cinématographique matérialisée par le changement de plan. Ce passage s'effectue en douceur grâce à une transition qui s'établit sur trois niveaux : celui des paroles, celui du bruitage et celui de l'image. Au niveau de la chaîne parlée :«Who comes here ?» demande Helena; puis, ayant reconnu Parolles, elle ajoute :

One that goes with him; I love him for his sake, And yet I know him a notorious liar.

I.i. $96-98$.

Ainsi' se trouvent liés le thème «Bertram» (le premier «him») et le thème «Parolles»; au niveau du bruitage : un bruit de pas signale d'abord à Helena l'arrivée d'un personnage, l'identification de Parolles ne vient qu'après; au niveau des images : l'acte de reconnaissance s'effectue grâce au miroir qui renvoie à Helena l'image du nouvel 
arrivant. Cela posé, venons-en à l'analyse du plan 1 .

Plan 1:

La dernière image précédant notre séquence livre un aperçu fugitif sur une enfilade de pièces, celles-là même que Bertram et Lafew vont traverser pour sortir. Le plan 1 commence sur une image qui montre, elle aussi, une enfilade de pièces mais dont on devine qu'elles se situent à l'opposé de la direction prise par les deux personnages. L'effet de cette juxtaposition d'images au contenu presque similaire est double : former la jointure entre les deux séquences et manifester le rapport de contiguité et, pourtant, d'altérité entre deux espaces : l'espace des adieux et l'espace du monologue, l'espace de la présence physique de Bertram et l'espace de la solitude d'Helena, lequel, par le pouvoir évocateur du monologue (au moins jusqu'à l'arrivée de Parolles) va devenir l'espace de la présence imaginée, rêvée, de l'être cher. Cette continuité spatiale qui restitue visuellement la continuité thématique autour du motif «Bertram» autorise une ellipse dans le déroulement chronologique des actions : on ne voit pas Helena passer dans la pièce à côté et s'asseoir au clavier; solution élégante qui fait l'économie d'une image-cheville à faible potentialité signifiante.

A cette continuité spatiale se superpose une continuité musicale. Une note de musique jouée sur un instrument qu'on devine être une épinette accompagne le départ de Bertram et de Lafew à la fin de la séquence précédente; or ce qui, de prime abord, pouvait passer pour une simple ponctuation sonore (imputable au cinéaste) ou un ornement extra-diégétique, est en réalité un élément anticipé de la diégèse, une préfiguration de l'épisode suivant où l'on voit Helena jouer de son instrument. Cette transition musicale opérée par un décrochement du son et de l'image est évidemment porteuse de significations connotées; tout se passe comme si la musique commence avec le désir de musique, désir éprouvé par Helena alors même qu'elle n'a pas encore quitté l'espace des adieux; d'où cette note jouée d'on ne sait quel lieu, note fantas- 
matique, inaudible pour les participants à l'action mais perçue par le téléspectateur; expression fugace d'un désir de musique en quoi il faut lire un désir de rêverie et de mélancolie amoureuse qui se concrétisera dans l'image suivante, quand d'autres notes bien «réelles» (puisque étayées par l'image) viendront relayer cette note «fictive».

L'image qui accompagne la partie musicale et les premières paroles d'Helena vise avant tout à situer le personnage dans l'espace. Le cadrage ne privilégie aucunement l'héroine. Lentement la caméra exécute un travelling-avant bientôt associé à un mouvement tournant qui prend le personnage à revers et s'immobilise sur le profil d'Helena filmé en plan américain (le buste et la tête). On remarque que l'arrêt de ce mouvement enveloppant coincide, au niveau des paroles, avec le constat brutal : «I am undone». Mais à quoi vise ce mode de filmage qui utilise aussi largement les mouvements d'appareil? D'abord à valoriser l'espace ambiant qui paraît, sinon plus vaste, du moins plus tangible en sa continuité, que s'il était filmé par plans successifs. La profondeur du champ, les contrejours, le dallage géométrique accentuent l'effet de perspective tandis que le reflet dans le miroir placé au-dessus de l'épinette renvoie, par métonymie, à l'espace de la salle. Mais cette emphase dénotative s'assortit également d'une intention connotative d'ordre psychologique et esthétique. Il s'agit, en premier lieu, de connoter le vide en dénotant l'espace; le vide des lieux, homologue du vide que ressent Helena après le départ de Bertram. Toutefois, l'environnement n'est pas présenté comme hostile ou même inconfortable; les mouvements d'appareil, on l'a dit, familiarisent le téléspectateur avec l'espace et les objets qui le meublent; la caméra joue le rôle d'un guide qui nous ferait visiter des lieux propres à susciter un plaisir esthétique. Nous touchons ici à l'autre versant de la connotation qui renvoie à la peinture : on se croit dans un intérieur hollandais peint par Pieter de Hooch ou Vermeer. Ce n'est pas par hasard que les objets disposés sur le guéridon, près de la fenêtre, composent une nature morte. 
Ceux qui ont vu le film entier ont pu observer que la tendance à connoter la peinture s'exprime en plusieurs endroits de la chaîne imagée, au point que l'ensemble de ces images picturales constitue un syntagme suprasegmental qu'il faudrait étudier en lui-même et dans ses rapports avec les autres syntagmes du texte filmique.

Si l'on considère maintenant la chaîne parlée, on remarque que la manière dont les paroles sont restituées à l'écran est, elle aussi, riche de connotations. Les trois premiers vers du monologue ont une résonance acoustique particulière, obtenue par le procédé de la voix off. Il s'agit du suggérer auditivement le caractère tout à fait intériorisé de ce début de monologue, ce que l'image ne tarde pas à confirmer : Helena se tait tout le temps qu'elle joue; outre que le timbre de la voix s'accorde avec l'impression de vide communiquée par l'image, l'intériorisation du monologue fait ressortir combien l'aveu d'Helena recèle d'inavouable, combien il est peu compatible avec les conditions ordinaires de la communication orale soumise à la censure des convenances :

$\mathrm{O}$, were that all! I think not on my father, And these great tears grace his remembrance

more

Than those I shed for him.

I.i. 77-79.

Après quoi, le timbre de la voix change; d'absente et de quasi irréelle, la voix devient plus présente, plus proche. Comme pour la musique, un discours oralisé vient relayer un autre discours, secret, intérieur, confidentiel. Quant à la pause musicale qui interrompt le monologue, laquelle d'ailleurs n'a rien de spécifiquement cinématographique (le théâtre opère de semblables manipulations), elle a pour fonction de souligner, autrement que par les mots, la structure thématique du discours; c'est le prélude musical à un thème nouveau : le portrait physique de Bertram et l'attirance qu'il exerce sur Helena.

Avant la fin de cet intermède musical, un travelling optique rapproche le visage d'Helena pour s'immobiliser 
une nouvelle fois, sur un constat négatif :«But now he's gone». Avec la reprise des paroles, se font entendre les premiers bruits de la séquence : des croassements de corbeaux rappellent, discrètement mais sûrement, à la fois la proximité vivante d'une nature champêtre et la réalité du moment présent en ce qu'il a de quotidien et de familier. Sur le fond de ce bruitage se détachent des paroles évocatrices du passé, mais d'un passé revécu dans sa dimension quotidienne et familière :

'Twas pretty, though a plague,

To see him every hour; to sit and draw

His arched brows, his hawking eye, his curls, In our heart's table.

I.i. $90-93$

Que penser du bruit de chevaux qui semble redoubler le constat «But now he's gone»? S'agit-il d'un simple pléonasme ? Je ne le pense pas. En faisant coïncider le départ de Bertram avec le moment présent, le réalisateur modifie la fonction et le sens de l'énoncé au sein du monologue. Ce qui pouvait apparaître comme l'articulation rhétorique d'un discours fondé sur l'opposition entre la présence imaginée de l'être aimé et son absence réelle devient l'expression spontanée d'un constat de fait, induit par la perception auditive du départ. Cette actualisation du moment de la séparation donne aux paroles un tour moins discursif, plus «naturel». L'effet dramatique n'en souffre pas : c'est comme si Bertram partait pour la seconde fois; départ invisible, certes, mais ressenti par Helena avec d'autant plus d'acuité qu'il survient au terme d'une méditation amoureuse. Toujours à propos du rapport entre le bruit et la parole, on observera que la question d'Helena : Who comes here ?» est précédée d'un bruit de pas qui, plus que la parole et avant celle-ci, signale une présence étrangère; préséance du bruit qui fait de la parole habituellement détentrice de l'effet dramatique un adjuvant du bruitage. On reviendra, au cours de l'analyse du deuxième segment filmique, sur la «dévaluation»de la parole théâtrale dans le film. 
Avant de passer au plan 2 de notre séquence, arrêtons-nous un instant sur le cadrage qui isole le visage d'Helena silhouetté sur le rectangle lumineux de la fenêtre. L'image exploite les possibilités connotatives de l'opposition du clair et du sombre; le clair c'est la partie du visage offerte au monde extérieur mais dérobée au téléspectateur; le sombre c'est l'autre partie du visage orientée vers le téléspectateur promu confident des pensées et des désirs secrets de l'héroine; procédé typiquement cinématographique qui exprime par l'image un rapport de connivence entre le téléspectateur et Helena. Lors du deuxième monologue à la fin de la scène, le visage de la jeune fille sera éclairé de face : le temps de la confession introspective est passé; la réflexion est, à présent, tournée vers l'action (l'élaboration du stratagème), laquelle conduira Helena à affronter en pleine lumière le regard et le jugement des autres personnages.

Plan 2:

C'est la première apparition de Parolles; l'image se subdivise en deux représentations métonymiques : le visage et le haut du buste reflétés dans le miroir, le bord externe du bras filmé en vision très rapprochée. Cette présentation fragmentaire et dédoublée isole mieux qu'une vision d'ensemble le détail pertinent, à savoir la coquetterie et la fatuité vestimentaires du personnage. Si la présence physique de Parolles est attestée par le flot de rubans cousu sur la manche, son identité en tant que personne n'est saisie que sous la forme médiatisée d'un reflet. La portée symbolique de cette présentation est claire : la réalité concrète du personnage est d'ordre vestimentaire (Lafew dit: $\ll$ The soul of this man is in his clothes», II.v. 43-44); quant à sa personnalité, elle a l'inconsistance et l'immatérialité d'une apparence spéculaire.

On remarquera qu'il n'y a aucune redondance entre le signifié visuel et le signifié verbal puisque le commentaire d'Helena concerne l'aspect moral du personnage :

I know him a notorious liar, 
Think him a great way fool, solely a coward.

I.i. 98-99 .

La parole chemine donc parallèlement à l'image sans la redoubler, jusqu'au moment où ces deux parallèles se recoupent au point précis où le monologue fait appel à la métaphore du vêtement :

Yet these fix'd evils sit so fit in him

That they take place when virtue's steely

bones

Look bleak i'th' cold wind.

I.i. 100-102.

Afin de rendre complètement vraisemblable la question que Parolles posera tout à l'heure à Helena :«Are you meditating on virginity ? $\rangle^{9}$ le réalisateur abandonne la convention théâtrale du monologue parlé pour celle, beaucoup plus cinématographique, du monologue en voix off; mais on ne retrouve pas ici l'effet acoustique noté précédemment, car ce qu'Helena dit de Parolles n'engage pas ses sentiments en ce qu'ils ont de plus profond.

Un mouvement d'appareil accompagne la sortie de Parolles après qu'un déplacement du personnage devant l'objectif eut momentanément rempli la presque totalité de l'image; la séquence s'achève sur une vue fixe analogue à la vue initiale, c'est-à-dire cadrée sur l'enfilade des pièces; souci de symétrie de la part du cinéaste ? C'est possible. Quoi qu'il en soit l'absence provisoire de Parolles se justifie doublement : c'est une fausse sortie qui prépare la véritable entrée du personnage, quand il réapparaîtra muni de son chapeau, accessoire indispensable de la conversation. D'autre part, cette absence coincide avec la conclusion du monologue d'Helena :

Withal, full oft we see

Cold wisdom waiting on superfluous folly

I.i. 102-103. Bien que «superfluous folly» se réfère encore à la vanité $\mathrm{du}$ vêtement, le jugement qu'expriment ces deux vers a une signification générale qui dépasse le cas de Parolles; il n'a donc nul besoin de s'appuyer sur un référent imagé; 
mieux, l'exclusion de tout référent particulier valorise la portée universelle de cette maxime en vers rimés.

\section{Etude du deuxième segment filmique : l'entrée du roi et d'Helena(II.iii. 39-51, éd. Arden).}

La séquence ne comporte pas moins de dix plans que je recense rapidement en me limitant d'abord aux images : - le plan 1 montre une enfilade de pièces dans le palais royal. La caméra est fixe et la profondeur de champ se trouve accentuée par le filmage en vue rapprochée de Lafew et d'un gentilhomme dont nous avons une vue partielle prise de dos.

- le plan 2 est une vue rapprochée du couple dansant; un mouvement d'appareil accompagne le passage latéral des deux personnages devant l'objectif. On peut croire, à ce stade, que la caméra restitue le point de vue des personnages-témoins. Il n'en est rien.

- le plan 3 est un plan fixe sur les quatre témoins filmés en vue rapprochée.

- le plan 4 est comme une réédition du plan 1 avec une profondeur de champ moindre. C'est cette image qui représente le point de vue des personnages-témoins comme le suggère le voisinage des plans 3 et 4 . Le roi et Helena continuent, leur avancée jusqu'au moment où, virant sur la gauche, ils quittent le champ de l'image.

- le plan 5 est un plan fixe sur les quatre personnagestémoins qui font la révérence au passage du roi.

- le plan 6 est un gros plan sur le visage de Bertram.

- le plan 7 est un plan général fixe sur une salle du palais. Le roi et Helena filmés de dos gagnent en dansant le centre de la pièce rejoints bientôt par le gentilhomme et quelques autres; c'est le moment où le roi, vu de face et cadré au centre de l'image, ordonne qu'on fasse venir les lords. La danse reprend de plus belle.

- le plan 8 est un plan fixe sur la danse filmée à travers une lucarne ovale; c'est une vue en plongée, sensiblement plus longue que les précédentes; le roi quitte sa cavalière, la 
reprend et voici qu'intervient un mouvement d'appareil descendant bientôt associé à un travelling optique qui s'immobilise sur l'image du roi vu de dos et s'asseyant face aux courtisans. On apporte un fauteuil pour Helena.

- le plan 9 est un plan fixe qui montre Helena s'installant auprès du roi.

- le plan 10 est un plan fixe plus éloigné; les lords entrent escortés par le gentilhomme.

L'intérêt de ce recensement fastidieux est de montrer combien le mode de narration diffère de celui étudié dans notre première séquence. La narration est ici fondée sur une extrême fragmentation de la chaîne imagée, au moins pendant la première partie de la séquence. Exception faite des plans 8,9 et 10 , on a une série discontinue d'instantanés dont la cohérence d'ensemble est assurée par la continuité temporelle de deux motifs diégétiques : la danse du roi et l'activité d'observation des personnagestémoins. Deux syntagmes distincts composent la séquence : un syntagme alterné du type : image des danseurs, image des témoins, image des danseurs etc. (du plan 1 au plan 6 inclus), et un syntagme linéaire comportant une seule série d'images, celles relatives aux faits et gestes du roi, au nombre desquelles il faut inclure l'arrivée des lords. Le montage alterné qui joue sur le rapport de simultanéité entre deux séries d'images consécutives est un élément primordial de la différence entre théâtre et cinéma. Si sophistiquées que soient les techniques d'éclairage, si vastes que soient les plateaux de théâtre autorisant une pluralité des aires de jeu et un échelonnement des plans, le thêâtre ne peut jamais obtenir une pareille fragmentation de la vision. Même éclaté dans l'espace, le spectacle de théâtre reste gagé sur la permanence matérielle du lieu scénique, intégralement offert aux déplacements du regard. Le metteur en scène monte un spectacle; le réalisateur de film monte des images.

S'agissant des paroles, qu'observe-t-on ? La séquence comporte à peine une douzaine de lignes dont sept vers alloués au roi. Au total, peu de paroles réparties sur un 
Du Texte à la Scène : Langages du Théâtre.

texte filmique émietté en dix plans distincts; surtout si l'on considère que les quatre derniers vers prononcés par le roi forment un bloc parlé qui occupe un seul plan, le plan 9 :

And with this healthful hand, whose banish'd sense

Thou hast repeal'd, a second time receive

The confirmation of thy promis'd gift,

Which but attends thy naming.

II.iii. 48-51. Un exemple fera mieux ressortir la fragmentation de la chaîne parlée. Dans le texte shakespearien la réplique de Lafew se lit:

Lustique, as the Dutchman says. I'll like a maid the better whilst I have a tooth in my head.

Why, he's able to lead her a coranto.

II.iii. $41-43$.

Trois propositions syntaxiquement indépendantes et sémantiquement autonomes; trois énoncés plutôt juxtaposés que reliés entre eux par un fil discursif. Que voit-on à l'écran ? «Lustique, as the Dutchman says» appartient au plan 1; aucune parole pour le plan 2; «I'll like a maid ... head» appartient au plan 3 ; «Why , he is able ... coranto» appartient au plan 4 . La fragmentation des images porte à un très haut degré la discontinuité du discours perceptible au niveau du texte.

Dans une comparaison entre le théâtre et le cinéma, Christian Metz note qu'au théâtre la parole est reine : «La parole théâtrale est Verbe parce qu'elle est souveraine et qu'elle crée la diégèse; la parole cinématographique n'est pas Verbe parce qu'elle est sujette et qu'elle fait partie de la diégèse, monde fictif quasi réel, qui se nourrit avant tout de la richesse des images $\rangle^{10}$. Ici la parole est encore théâtrale - puisque shakespearienne - mais l'usage qui en est fait la rapproche du statut moyen de la parole cinématographique; je dis «statut moyen» car le film peut inclure des énoncés dramatiques («mots» de théâtre, réparties, dialogues de scène, récits monologués etc .). 
Mais, en règle générale, la parole cinématographique ou télévisuelle est, comme le dit Metz, plus proche de la parole réelle de la vie que de la parole vraie du théâtre; elle tend même à devenir parfois ce qu'Albert Laffay appelle la «rumeur du personnage», sorte de «bruitage humain» (Ch. Metz) qui l'apparente à la parole quotidienne et l'éloigne de la parole stylisée, épurée, concentrée du théâtre. Même la dédramatisation de la parole à laquelle s'efforce Harold Pinter est encore une esthétique de théâtre. Ici les paroles sont à demi immergées dans les images, lesquelles, du même coup, retrouvent leur vocation cinématographique qui est de cimenter le discours filmique. Les paroles sont aussi à demi immergées dans la musique, musique de fond (ou d'accompagnement) qui soutient la danse mais qui, contrairement à la mélodie jouée par Helena, n'intervient pas dans le cours de la narration filmique. Des trois eléments qui constituent la bande sonore (musique, paroles, bruits), la musique est ici l'élément le moins signifiant, moins signifiant que les bruits, c'est-à-dire les exclamations qui couvrent les plans 2 et 4 et se prolongent en murmures pendant toute la durée de la séquence; ces rumeurs collectives dénotent la réjouissance et l'émerveillement du public face à la guérison miraculeuse du roi; elles connotent le consensus établi autour de la personne royale et de la figure salvatrice d'Helena; de sorte que le gros plan sur le visage grave et songeur de Bertram retranche le personnage de la collectivité et le projette dans l'isolement du héros ${ }^{11}$.

On ne peut conclure l'examen de cette séquence sans dire un mot du plan 8 (le plan de la lucarne) qui frappe par son caractère singulier. Pourquoi cette vue plongeante sur le roi dansant, filmé à travers un oeil-de-boeuf ? On peut penser que ce choix ne fait que renvoyer à l'habituel parti-pris de produire des images picturales, d'où ce petit tableau vivant inscrit dans un médaillon. Mais les images picturales du film se présentent autrement; comme les autres images, elles se donnent pour des représentations directes de la réalité ; c'est la réalité qui est aménagée de 
façon à suggérer la peinture (choix des couleủrs, composition, effets de lumière, statisme relatif du sujet etc...). Or ici, l'effet de peinture est obtenu par un aménagement de la vision : c'est uniquement la présence du cadre ovale qui fait penser à un tableau. Ici l'effet de peinture dépend de la perception et non de l'objet perçu; perception tout d'abord médiate (et cadrée) : la scène est vue à travers une lucarne qui délimite le champ visuel, puis immédiate et «libre»: un mouvement d'appareil descendant nous restitue la vision directe de l'objet. Ce qui est intéressant n'est donc pas tant l'existence de cette vue en médaillon que le passage d'une perception médiate à une perception immédiate, ce qui nous oriente vers un sens connoté autre que pictural. L'effet combiné de l'image fixe et du mouvement d'appareil est de suggérer, par un procédé spécifiquement cinématographique, le proche et le lointain $^{12}$; montrer combien le spectacle incongru d'un roi en chemise et qui danse est pour nous chose lointaine : le médaillon, jusque dans sa forme même, projette le téléspectateur dans l'univers magique et fabuleux du conte illustré; montrer aussi quelle frontière ténue sépare le lointain du proche : il suffit qu'un autre regard redescende au niveau de l'objet pour que le lointain redevienne proche et que le spectateur, quittant l'univers du fabuleux, se retrouve de plain-pied avec l'univers de la fable.

Il est impossible, au terme d'une analyse aussi fragmentaire, de conclure quoi que ce soit sur la «valeur» de l'adaptation télévisée d'All's Well. Ce n'était d'ailleurs pas le but recherché. Le réalisateur de cinéma ou de télévision qui prend pour script un texte qui préexiste à la fabrication du film (roman, nouvelle, texte de théâtre, poème etc.) se trouve dans une situation comparable à celle du metteur en scène; comme lui, il est appelé à découper dans la polysémie du texte; comme lui, il doit opérer des choix qui, nécessairement, privilégient certaines significations et en occultent d'autres; comme lui, il préside à un travail d'accouchement du sens au terme duquel voient le jour des significations qui n'avaient dans le texte 
qu'une existence encore purement virtuelle. Toute mise à l'écran (comme toute mise en scène) est un discours sur un texte, discours au travers duquel l'artiste exprime avec plus ou moins de cohérence et quelle que soit la conscience qu'il aît de tenir un discours - sa position vis-à-vis du texte, du public, de son art, et des autres discours, contemporains ou antérieurs, tenus sur le même texte.

La tâche du sémioticien du film est complexe; elle se situe à trois niveaux étroitement imbriqués dans la réalité mais dissociables théoriquement; au niveau du langage, le sémioticien s'emploie à démêler et à décrire le fonctionnement des codes cinématographiques (montage, mouvements d'appareil, échelonnement des plans, cadrage, effets optiques etc.) dont la mise en oeuvre fait du film un mode spécifique d'expression et de communication; au niveau du texte filmique où l'on a toujours affaire à des textes singuliers, le travail du sémioticien consiste à exhiber les mécanismes d'une écriture filmique particulière, que ce soit au niveau du film entier (ou d'un corpus de films) ou au niveau de la séquence, voire du plan; au niveau du discours, le texte filmique est considéré comme un ensemble construit de messages signifiants générés en fonction de deux compétences articulées l'une à l'autre : une compétence proprement cinématographique et une compétence plus générale, d'ordre idéologique ${ }^{13}$. Dans cette perpective, la tâche du sémioticien est de décrire les processus d'engendrement des significations.

Si la microanalyse à laquelle je me suis livré ne débouche sur aucune conclusion applicable à la totalité du téléfilm All's Well That Ends Well, si elle ne permet pas non plus de saisir la spécificité du langage télévisuel par rapport au langage proprement cinématographique, elle aura, du moins, mis en évidence l'imbrication des trois niveau $x$ que je viens de rappeler. Ce travail n'a évidemment d'autre ambition que de servir de matériau pour une future sémiologie du spectacle dramatique télévisuel. 


\section{NOTES}

1. Le cinéma a de solides références shakespeariennes : Henry $V$ t Richard III de Lawrence Olivier, Macbeth et Othello d'Orson Welles, Hamlet et Le roi Lear de Kozintsev etc.

2. Christian Metz se fait l'écho de ce mépris quand il évoque les «méchantes bandes du «théâtre filmé»par opposition au "théâtre ciném atographique» (Essats sur la signification au cinéma, tome 2, ed. Klincksiek, 1972, ed. 1981, p. 51). S'il est évident pour tout le monde que ces films sont souvent assez insipides, dun point de vue strictement cinématographique, il reste que dans la mise au service du théâtre des moyens techniques dont ils disposent, certains réalisateurs peuvent accomplir un excellent travail; les retransmissions télévisées du $R$ oi Lear $m$ is en scène par Daniel Mesguisch et de Peines d'amour perdues $m$ is en scène par JeanPierre Vincent nous ont valu de très beaux moments de théatre filmé indépendamment de la qualité du travail de $m$ ise en scène.

3. L'expression est de Georges Sadoul, citée par Ch. Metz, ibid.p. 63 .

4. Sur cette question on se reportera utilement à «l'entretien sur la sémiologie du cinéma »(1970) entre Ch.Metzet R.Bellour, qui conclut le tome 2 des Essais sur la signification au cinéma, op. cit., pp. 196-219.

5. Ch. Metz : Langage et cinéma, ed. Larousse, $1971, \mathrm{p} .180$.

6. On appelle «texte filmique» non pas la couche tex tuelle du film (la série des paroles) mais la contexture des signes appartenant aux cinq charnes constitutives du texte filmique, lesquels sont différents de par leur substance (linguistique, musicale, imagée etc ...) mais peuvent remplir des fonctions signifiantes analogues. Mutatis mutandis, rien n'empêche de parler, à propos du théâtre, de «texte scénique»pour le distinguer du tex te verbal.

7. On se réfère ici à la définition du plan la plus courante en filmologie: le plan est l'ensemble des images résultant d'une prise de vue ininterrompue, d'un seultenant.

8. Dans la nomenclature proposée par Ch. Metz la séquence au sens restreint du mot se définit par quatre caractères qui s'énoncent ainsi, par ordre croissant de particularisation : syntagme chronologique (par opposition au $x$ syntagmes a-chronologiques), narratif (par opposition à descriptif), linéaire (par opposition à alterné), à consécution discontinue (par opposition à la «scène» au sens précis du terme qui, elle, présente une consécution continue)

9. G'est moi qui souligne. Un mot de plus sur la question que Parolles pose à Helena, assise à l'épinette; si l'on se souvient que cet instrument est également afpelé "virginal» (surtout en Angleterre), on a ici un trait desprit (virginal/virginity) non prévu par Shakespeare mais imputable au réalisateur du film. Ce n'est ni 
très fin, ni très délicat, juste ce qu'il faut pour Parolles.

10. Ch. Metz : Essais sur la signification au cinéma, tome 2, op. cit., p. 53 .

11. Ce qui ne veut $p$ as dire que Bertram est «héroique». II appartienđrait plutôt à la catégorie des héros «négatifs».

12. J'emprunte les deux termes de cette dialectique féconde à Richard Marienstras : Le proche et le lointain, ed. de Minuit, 1981.

13. On définira la compétence ciném atographique comme le système (intériorisé $p$ ar le sujet filmant) des règles qui régissent la production d'un film. La compétence idéologique est le système de représentation en fonction duquel sont produites les significations; la performance (la réalisation du film sous ses deux aspects, comme tex te et discours) est $1 \mathrm{a} \mathrm{m}$ ise en oeuvre de cette double compétence. A propos de l'utilisation possible du concept de compétence idéologique appliqué à l'étude des significations d'un texte dramatique, on pourra se reporter à mon article : «Lecture idéologique d'un tex te de théâtre : The Changeling» in Aspects du Théâtre anglosaxon, Publications de l'Université de Rouen, $1981, \mathrm{pp} .17-43$. 